\title{
ESCLERITIS NODULAR POSTERIOR GIGANTE COMPATIBLE CON SARCOIDOSIS OCULAR SIMULANDO UN MELANOMA DE LA COROIDES
}

\section{GIANT NODULAR POSTERIOR SCLERITIS COMPATIBLE WITH OCULAR SARCOIDOSIS SIMULATING CHOROIDAL MELANOMA}

\author{
PÉREZ-CAMPAGNE E ${ }^{1}$, GUEX-CROSIER Y², SCHALENBOURG A ${ }^{2}$, UFFER S ${ }^{2}$, ZOGRAFOS L $^{2}$
}

\section{RESUMEN}

Caso clínico: Un paciente de 30 años fue enviado a nuestro servicio oncológico de referencia con el diagnóstico de melanoma amelanótico de la coroides del ojo izquierdo. Se hicieron los siguientes exámenes: oftalmoscopía, angiografía fluoresceínica, angiografía con verde de indocianina, ecografía, resonancia magnética y biopsia.

Discusión: El diagnóstico de escleritis nodular posterior gigante basado en la ecografía se confirmó mediante una biopsia. Se realizó un examen médico completo buscándose el posible agente causal; no se encontró la etiología. La histología reveló un granuloma que era compatible con una sarcoidosis ocular. Mediante la administración de esteroides por vía oral $(1 \mathrm{mg} / \mathrm{kg})$, se obtuvo una evolución rápidamente favorable del cuadro. El diagnóstico de esta afección sigue siendo un desafío. Es importante diferenciar una escleritis posterior nodular de un melanoma de la coroides.

Palabras clave: Escleritis, tumores del ojo, tumores de la úvea, tumores de la coroides, sarcoidosis, melanoma de la coroides.

\begin{abstract}
Case report: A 30-year-old man was referred to our ocular oncology service with a diagnosis of amelanotic choroidal melanoma of the left eye. The following tests were performed: ophthalmoscopy, fluorescein angiography, indocyanine green angiography, ultrasonography, magnetic resonance imaging and biopsy.

Discussion: The diagnosis of giant nodular posterior scleritis, as suggested by ultrasonography, was confirmed by biopsy. A comprehensive medical evaluation was performed, but no etiology was found. The histology revealed a granuloma compatible with ocular sarcoidosis. A rapid response was obtained by systemic steroid administration $(1 \mathrm{mg} / \mathrm{kg})$. Sarcoidosis continues to be a challenge in diagnosis. It is important to distinguish nodular posterior scleritis from choroidal melanoma (Arch Soc Esp Oftalmol 2007; 82: 563-566).
\end{abstract}

Key words: Scleritis, eye neoplasms, uveal neoplasms, choroid neoplasms, sarcoidosis, choroidal melanoma.

\footnotetext{
Recibido: 31/5/06. Aceptado: 25/7/07.

Hôpital Ophtalmique Jules Gonin. Université de Lausanne. Lausanne (Suiza).

1 Licenciado en Medicina.

2 Doctor en Medicina.

Comunicación presentada en el LXXXII Congreso de la S.E.O. (La Coruña 2006).

Correspondencia:

Eric Pérez-Campagne

Hôpital Ophtalmique Jules Gonin

Avenue de France, 15

CH-1004 Lausanne

Suiza

E-mail: eperezcampagne@gmail.com
} 


\section{INTRODUCCIÓN}

La escleritis nodular posterior gigante es una patología inflamatoria severa de la esclera posterior que puede provocar un desprendimiento coroideo hasta la ora serrata. Representa el $2-12 \%$ de todos los casos de escleritis. Afecta más a las mujeres (66-83\%). Puede presentarse en una enfermedad granulomatosa, pero en la mayoría de los casos es idiopática y es unilateral (1). Dada su forma y volumen puede ser confudida con un tumor uveal $(2,3)$. Se presenta un caso que nos ha sido remitido como un melanoma de la coroides.

\section{CASO CLÍNICO}

Un varón caucásico de 30 años, sin antecedentes médicos significativos, fue enviado a nuestro servicio por un melanoma de la coroides en el ojo izquierdo (OI) en noviembre de 2005.

Desde agosto de 2005 el paciente percibía una disminución de la agudeza visual (AV) unilateral en el OI. Desde hace un mes se quejaba de dolores oculares en dicho ojo.

La AV del ojo derecho (OD) era de 10/10 con una corrección esférica de $-0,5 \mathrm{D}$, la del OI era $<1 / 10$ sin corrección y de 8/10 con corrección esférica sobreañadida de $-5 \mathrm{D}$.

El exámen oftalmológico del OD no mostraba ninguna particularidad.

El exámen a la lámpara de hendidura del OI mostraba una inyección ciliar marcada, una reacción inflamatoria leve en la cámara anterior y un iris abombado; la tensión ocular (TO) era de $17 \mathrm{mmHg}$. En el fondo del OI destacaba la presencia en el sector nasal de una masa amelanótica circunscrita (fig. 1A). La ecografía B demostraba un engrosamiento de la pared ocular que sobrepasaba los $5 \mathrm{~mm}$. En el espacio extraescleral se observaba una zona hipoecogénica (fig. 1B). Con la angiografía fluoresceínica y con verde de indocianina esta lesión estaba rodeada de pliegues coroideos bien visualizados; no se observaba una red vascular intratumoral propia (fig. 2).

Una resonancia magnética nuclear (RMN) orbito-cerebral confirmó el engrosamiento escleral de aspecto nodular que captaba el contraste en la zona postero-interna del OI, sin poner en evidencia otras lesiones (fig. 3).

Un profundo estudio en busca de una etiología reumática (factor reumatoide, Ac antinucleares, cANCA,

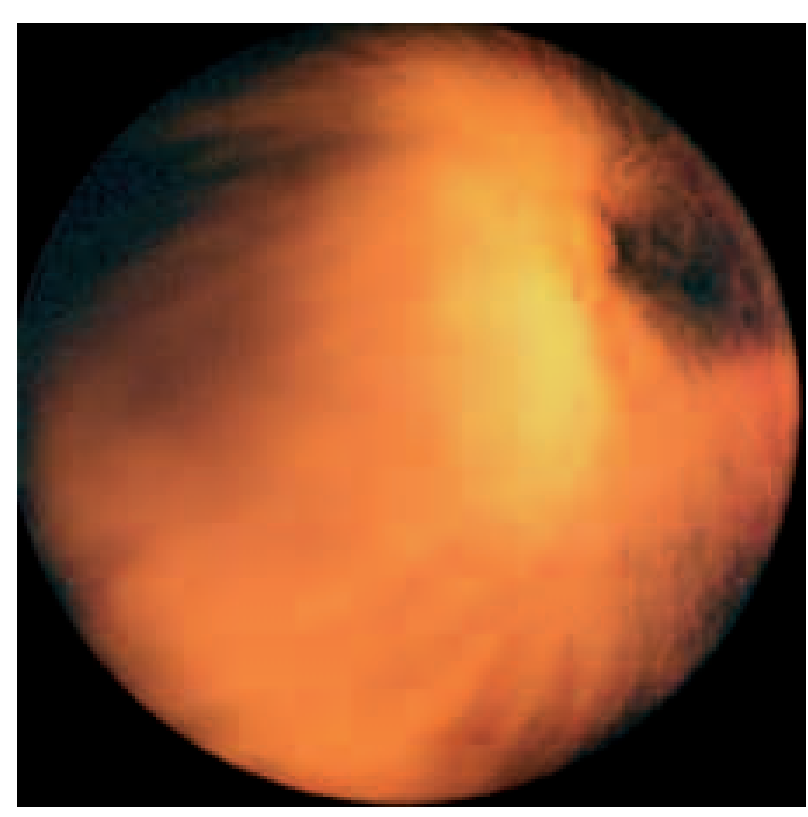

Fig. 1A: Fondo del OI. Presencia en el sector nasal de una masa amelanótica circunscrita.

Ac antifosfolípidos) y de una etiología infecciosa (TPHA, toxocarosis) resultó negativo. Los niveles de calcio en sangre fueron normales: $2,39 \mathrm{mmol} / \mathrm{l}$. La radiografía de tórax fue normal. La enzima de conversión de la angiotensina, discretamente aumentada:

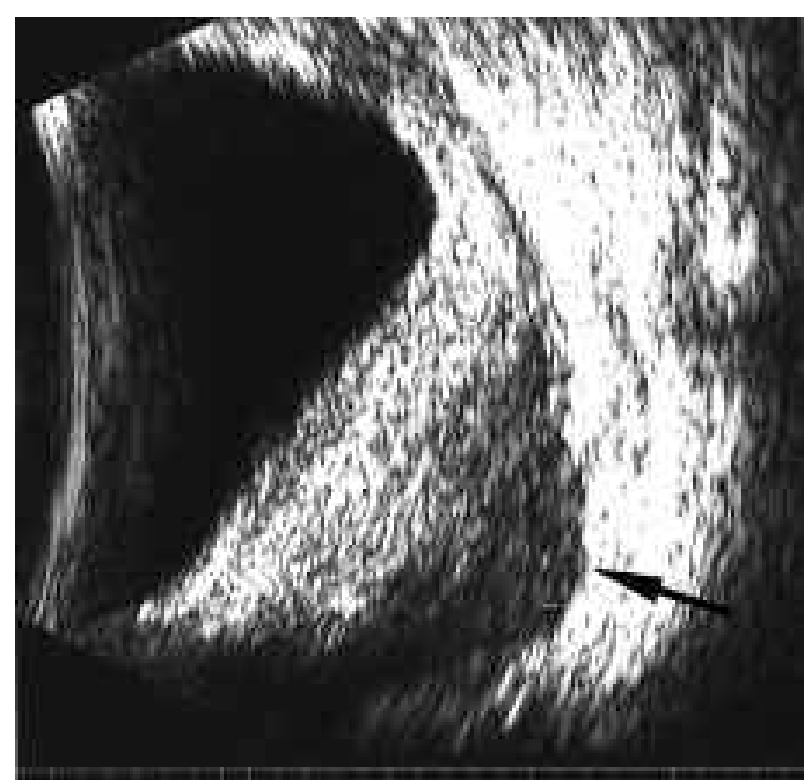

Fig. 1B: Ecografía B. Engrosamiento retinocoroideo y de la esclera que sobrepasa los $5 \mathrm{~mm}$. En el espacio extraescleral (flecha) se ve una zona hipoecogénica correspondiente a la inflamación del espacio subtenoniano. 

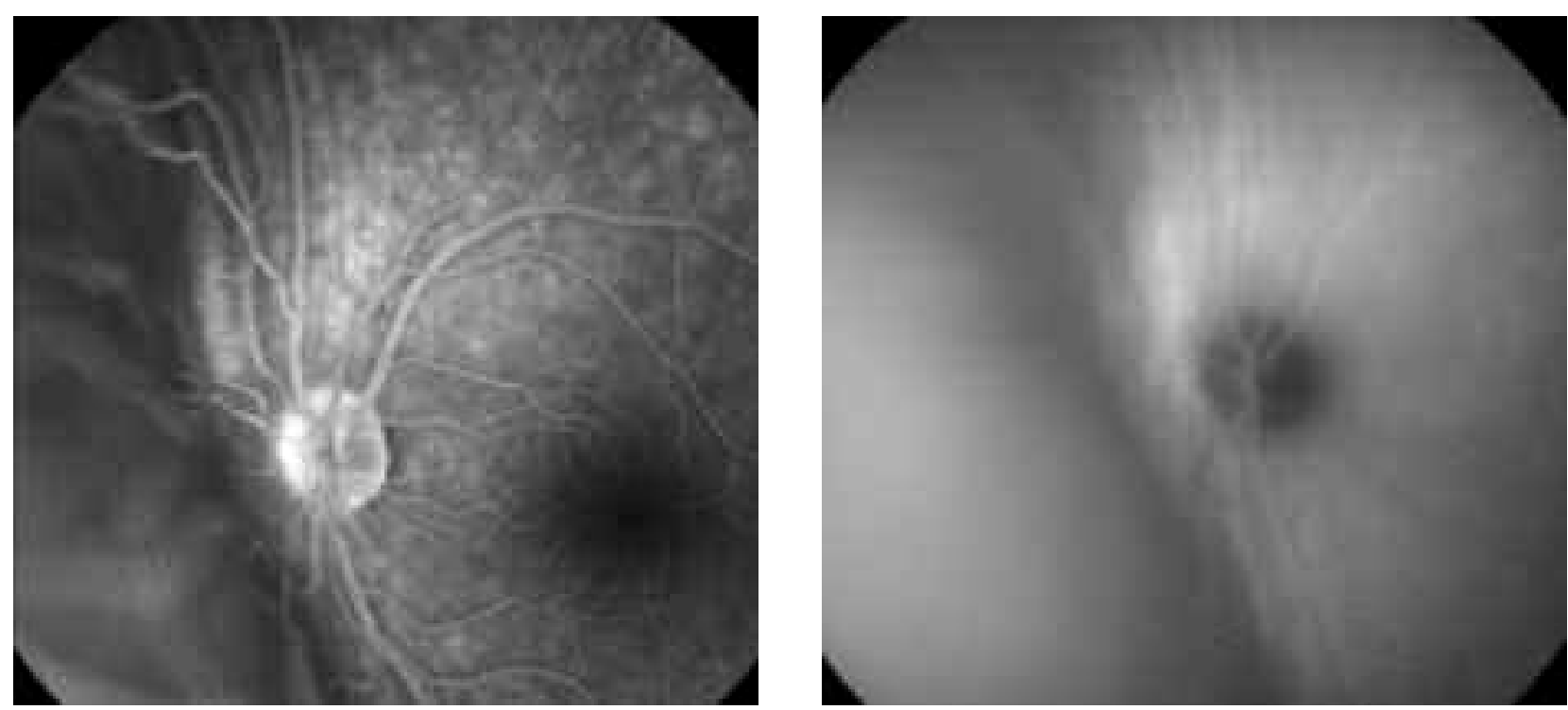

Fig. 2: Angiografía fluoresceínica (A) y con verde de indocianina (B). Presencia de los pliegues de la coroides en el borde de la masa. No se visualizaba una red vascular intratumoral propia.

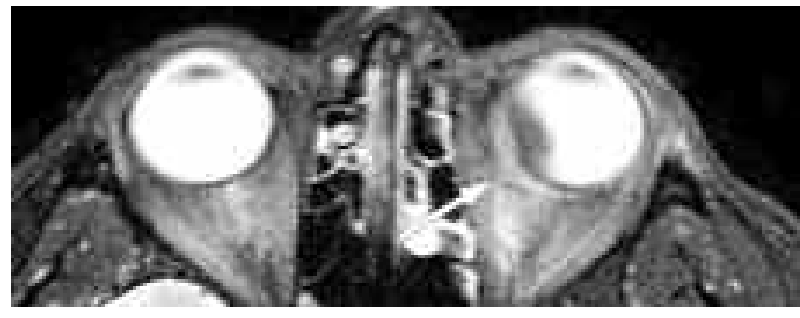

Fig. 3: RMN. Imagen axial T2 con supresión de grasa. El engrosamiento de la pared ocular es hipointenso con respecto al vítreo; la capa extra-escleral hiperintensa corresponde al líquido subtenoniano (flecha).

73U/L (normal, 10-55), era compatible con una afección granulomatosa, pero el resto de los exámenes no han permitido mostrar otros argumentos en favor de una sarcoidosis ni de una tuberculosis.

Teniendo en cuenta la anamnesis, el exámen clínico, angiográfico, ecográfico y la RMN se hizo el diagnóstico de escleritis posterior gigante. Se practicó una biopsia por vía extra-escleral y el resultado del examen histopalológico confirmó una escleritis granulomatosa compatible con una sarcoidosis (ausencia de agentes patógenos visibles con la coloración de Ziehl-Neelsen, Gram, metamina de plata y PAS) (fig. 4).

Se instauró un tratamiento por vía oral de corticoides, a dosis de $1 \mathrm{mg} / \mathrm{kg} /$ día rebajando progresivamente la dosis, debido a una buena respuesta terapéutica, a $0,5 \mathrm{mg} / \mathrm{kg} /$ día a los 6 meses de tratamiento. Se asoció una pauta local en el OI de dexa-

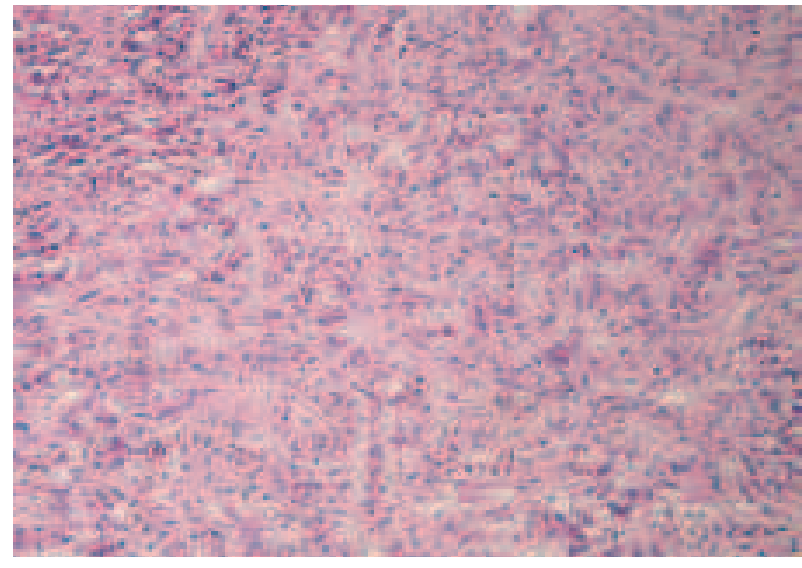

Fig. 4: Anatomía patológica. Hematoxilina-Eosina, x200. Granuloma constituido de células epitelioides, rodeado de algunos linfocitos.

metasona $0,1 \% 1$ gota 3 veces al día, para tratar la inflamación en la cámara anterior; y atropina 1\% 1 gota 3 veces al día, para disminuir el espasmo ciliar.

El examen de control del OI a los 6 meses mostró una AV de 10/10 con una corrección esférica de +0,50 D. Desapareció la inyección ciliar al igual que la reacción inflamatoria en cámara anterior, el cristalino estaba transparente; la TO era de $15 \mathrm{mmHg}$. En el fondo de ojo el levantamiento sólido en el sector nasal se aplanó (fig. 5A). En la ecografía B el espesor total de la pared ocular en el sector nasal inferior era de $2 \mathrm{~mm}$ aproximádamente (fig. 5B). 


\section{DISCUSIÓN}

Este caso ilustra el desafío del diagnóstico diferencial entre un melanoma de la coroides y la escleritis nodular posterior gigante.
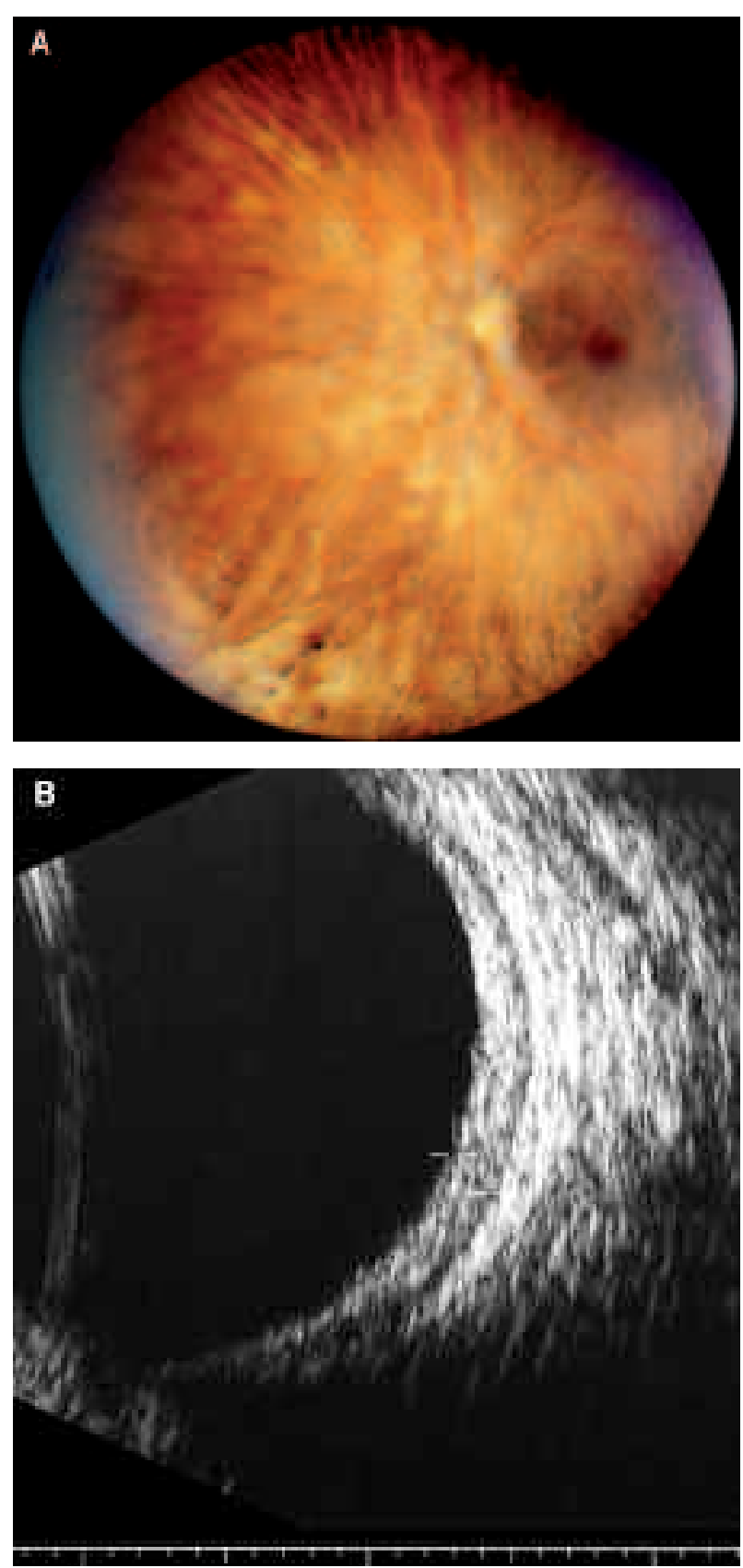

Fig. 5: Después de 6 meses de tratamiento con corticoides. A: Fondo del OI. Desaparición de la masa en el sector nasal. B: Ecografía B. Reducción del espesor de la lesión a $2 \mathrm{~mm}$ aproximádamente.
En la literatura, entre 1956 y 1973, diferentes estudios han hecho estadísticas sobre los diagnósticos histopatológicos en los globos oculares enucleados, consecuencia de un diagnóstico erróneo de un melanoma uveal; el porcentaje de las uveítis o esclerosis posterior varía entre 0,5 y $6,6 \%$. Existe una disminución de los errores diagnósticos a partir de la década de los 70 con la llegada de los medios diagnósticos modernos (4).

En este paciente varón diversos elementos nos dirigieron al diagnóstico de una escleritis posterior. El dolor ocular y la inyección ciliar eran compatibles con una patología inflamatoria provocando un espasmo ciliar y una miopía temporal. En el fondo del ojo una masa amelanótica importante asociada con pliegues coroideos sin red vascular propia hacen sugerir una escleritis posterior. La ecografía pone en evidencia un engrosamiento de la pared ocular asociado a la presencia de líquido extraescleral correspondiente a la inflamación del espacio subtenoniano; esto ha reforzado nuestra sospecha. La RMN aleja la eventualidad de un proceso expansivo, sin poder excluir un linfoma. Estos elementos fueron la base para realizar una biopsia escleral que ha confirmado el diagnóstico de escleritis nodular posterior gigante compatible con sarcoidosis. En las sarcoidosis oculares sólo el 25-50\% tiene una afectación sistémica (5). Es típica la excelente respuesta al tratamiento con corticoides.

\section{AGRADECIMIENTO}

A los señores Curchod y Winkler y a todo su equipo de fotografía del Hôpital Ophtalmique Jules Gonin de Lausanne.

\section{BIBLIOGRAFÍA}

1. Benson WE. Posterior scleritis. Surv Ophthalmol 1988; 32: 297-316.

2. Finger PT, Perry HD, Packer S, Erdey RA, Weisman GD, Sibony PA. Posterior scleritis as an intraocular tumour. Br J Ophthalmol 1990; 74: 121-122.

3. Demirci H, Shields CL, Honavar SG, Shields JA, Bardenstein DS. Long-term follow-up of giant nodular posterior scleritis simulating choroidal melanoma. Arch Ophtalmol 2000; 118: 1290-1292.

4. Zografos L. Tumeurs intraoculaires. París: Société Française d'Ophtalmologie; Masson; 2002; 172-175.

5. Ocampo VV, Foster CS, Baltatzis S. Surgical excision of iris nodules in management of sarcoid uveitis. Ophtalmology 2001; 108: 1296-1299. 